\title{
Efectos beneficiosos de los corticoides inhalatorios en los pacientes con enfermedad pulmonar obstructiva crónica
}

Multicentre randomised placebo-controlled trial of inhaled fluticasone in patients with cronic obstructive pulmonary disease.

Paggiaro P, Dahle R, Bakran I et al. The Lancet 1998;351:773-780

\section{Objetivo}

Evaluar la eficacia y la seguridad del propionato de fluticasona en pacientes con enfermedad pulmonar obstructiva crónica (EPOC).

Diseño

Ensayo clínico, aleatorizado, doble ciego y controlado con placebo.

Lugar

Estudio multicéntrico con pacientes ambulatorios reclutados en 13 países europeos, Sudáfrica y Nueva Zelandia.

\section{Pacientes}

Se aleatorizaron 281 pacientes con edades entre 50 a 75 años, con un volumen espiratorio forzado en el primer segundo (VEF1) del 35 al $90 \%$ del predicho, un VEF1 / capacidad vital forzada (CVF) $\leq 70 \%$, una reversibilidad de la obstrucción menor al 15\% luego de la inhalación de salbutamol y que no hubieran tenido cambios marcados en los últimos meses. Debían ser fumadores o ex fumadores de más de $10 \mathrm{pa-}$ quetes/año, cumplir criterios de bronquitis crónica y haber tenido por lo menos una exacerbación anual que requiriera consulta médica en los últimos tres años y una alta probabilidad de tener una exacerbación en los siguientes seis meses.

Fueron excluídos los pacientes con radiografía anormal, los que en las últimas cuatro semanas hubieran estado internados, hubieran recibido antibióticos, corticoides de depósito, fluticasona, o más de $500 \mu \mathrm{g}$ de otros corticoides inhalatorios. Se definió como exacerbación el empeoramiento de los síntomas que requiriera cambios en el tratamiento normal.

Durante dos semanas se suspendieron los corticoides inhalatorios (en quienes ya los recibían previamente) y los pacientes comenzaron a registrar en una cartilla la magnitud de la disnea, la tos y el volumen y color de las secreciones (cuantificados con una escala que variaba de 0 a 5 ).

Concluído este período, se aleatorizaron 281 pacientes en dos grupos de similares características. Un grupo recibió fluticasona $500 \mu \mathrm{g} / 12 \mathrm{~h}$ y el otro recibió placebo. Podían aliviar los síntomas con agonistas ß2 y no se alteraron las dosis de otras drogas que estuvieran recibiendo.

\section{Medición de resultados}

Se hizo el análisis por intención de tratar. El resultado principal que se midió fue el número de pacientes que tuvieron al menos una exacerbación durante el período de tratamiento. A las 4, 8, 16 y 24 semanas se midió la función pulmonar, la eficacia del tratamiento y la distancia que los pacientes pudieron caminar durante 6 min.

\section{Resultados principales}

\begin{tabular}{|c|c|c|c|c|}
\hline & Fluticasona & Placebo & $\begin{array}{l}\text { NNT } \\
(@)\end{array}$ & $p$ \\
\hline $\begin{array}{l}\text { Cantidad de pacientes que } \\
\text { tuvieron al menos una exacerbación }\end{array}$ & 32 & 37 & & $<0.067$ \\
\hline $\begin{array}{l}\text { Cantidad de internaciones } \\
\text { cada } 100 \text { pacientes }\end{array}$ & 2.81 & 9.35 & 15.29 & 0.02 \\
\hline $\begin{array}{l}\text { Cantidad de exacerbaciones } \\
\text { moderadas(\#) o severas( } \& \text { ) cada } \\
100 \text { pacientes tratados }\end{array}$ & 19 & 31 & 8 & $<0.001$ \\
\hline Disfonía & $4 \%$ & $1 \%$ & & $\begin{array}{l}\text { No } \\
\text { reportada }\end{array}$ \\
\hline Candidiasis & $3 \%$ & $1 \%$ & & \\
\hline $\begin{array}{l}\text { Infecciones respiratorias } \\
\text { altas }\end{array}$ & $13 \%$ & $10 \%$ & & \\
\hline $\begin{array}{l}\text { Concentración del cortisol sérico } \\
\text { inferior al normal en algún momento } \\
\text { del tratamiento }\end{array}$ & $13 \%$ & $11 \%$ & & $<0.024$ \\
\hline
\end{tabular}

(@) Número de pacientes que es necesario tratar para evitar un evento en ese período (datos calculados por el comité editorial).

(\#)Se definió como exacerbaciones moderadas a las que requirieron atención ambulatoria. (\&) Se definió como exacerbaciones severas a las que requirieron internación.

\section{Conclusiones}

El propionato de fluticasona puede ser beneficioso en pacientes con EPOC si es usado durante un mínimo de seis meses.

\section{COMENTARIO}

Como dicen los autores, ya estaba comprobado el beneficio de los corticoides inhalatorios en los pacientes asmáticos. Si bien es una práctica bastante frecuente su uso también en enfermos con enfermedad pulmonar obstructiva crónica, todavía no estaba demostrada totalmente su utilidad y era necesario contar con estudios controlados doble ciego como el que aquí comentamos.

Este trabajo no logró encontrar diferencias significativas en los resultados principales que quiso investigar (disminuir la cantidad de pacientes que tuvieran como mínimo una exacerbación durante ese período), pero encontró diferencias significativas en eventos clínicamente relevantes. El grupo tratado con fluticasona tuvo menor probabilidad de sufrir exacerbaciones moderadas y severas (definidas como las que requirieron atención ambulatoria o internación, respectivamente), necesitándose tratar 15 pacientes durante seis meses para evitar una internación.

Este estudio aporta mayor evidencia a favor de los beneficios de los corticoides inhalatorios en el tratamiento de la EPOC, sin embargo el tema no queda todavía cerrado. Siendo aquella una enfermedád crónica, serán necesarios trabajos con más tiempo de seguimiento para poder evaluar los efectos beneficiosos y adversos a largo plazo. Sin embargo es de suponer que, tratándose de pacientes con edades que oscilan entre 50 y 75 años, la probabilidad de que tengan suficiente tiempo para desarrollar los efectos adversos será baja.

\section{Dr. Sebastián Ferreiro \\ Sanatorio Anchorena. \\ Dr. Sergio Terrasa \\ Unidad de Medicina Familiar y Preventiva. Hospital Italiano de Buenos Aires.}

